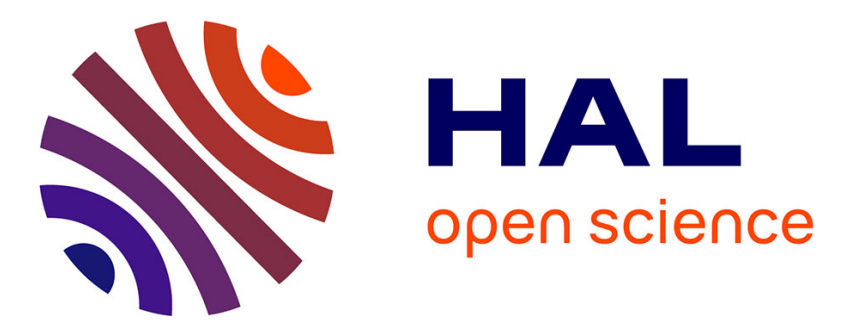

\title{
Determining the shape of defects in non-absorbing inhomogeneous media from far-field measurements
}

Yann Grisel, Vincent Mouysset, Pierre-Alain Mazet, Jean-Pierre Raymond

\section{To cite this version:}

Yann Grisel, Vincent Mouysset, Pierre-Alain Mazet, Jean-Pierre Raymond. Determining the shape of defects in non-absorbing inhomogeneous media from far-field measurements. Inverse Problems, 2012, 8 (5), pp.055003. 10.1088/0266-5611/28/5/055003 . hal-00805796

\section{HAL Id: hal-00805796 https://hal.science/hal-00805796}

Submitted on 28 Mar 2013

HAL is a multi-disciplinary open access archive for the deposit and dissemination of scientific research documents, whether they are published or not. The documents may come from teaching and research institutions in France or abroad, or from public or private research centers.
L'archive ouverte pluridisciplinaire HAL, est destinée au dépôt et à la diffusion de documents scientifiques de niveau recherche, publiés ou non, émanant des établissements d'enseignement et de recherche français ou étrangers, des laboratoires publics ou privés. 


\title{
Determining the shape of defects in non-absorbing inhomogeneous media from far-field measurements
}

\author{
Y. Grisel ${ }^{1}$, V. Mouysset ${ }^{1}$, P.-A. Mazet ${ }^{1}$ and J.-P. Raymond ${ }^{2}$ \\ 1 Onera - The French Aerospace Lab, F-31055 Toulouse, France \\ ${ }^{2}$ Université Paul Sabatier, Institut de Mathématiques de Toulouse, 31062 Toulouse \\ Cedex, France
}

\begin{abstract}
We consider non-absorbing inhomogeneous media represented by some refraction index. We have developed a method to reconstruct, from far-field measurements, the shape of the areas where the actual index differs from a reference index. Following the principle of the Factorization Method, we present a fast reconstruction algorithm relying on far field measurements and near field values, easily computed from the reference index. Our reconstruction result is illustrated by several numerical test cases.
\end{abstract}

2000 MSC: $35 \mathrm{P} 25,35 \mathrm{R} 30,35 \mathrm{R} 05,47 \mathrm{G} 40$

Acoustic scattering, Inhomogeneous media, Factorization method, Defect localization

\section{Introduction}

We consider an inverse scattering problem consisting in shape reconstruction from physical measurements. Since only specific parameters have to be determined, reconstruction can be expected to be faster than in the general case.

A family of shape identification methods from far-field measurements is represented by the Linear Sampling method [1, 2, 3]. This method is very fast, which makes it interesting from an applicative point of view. It consists in a pointwise binary test: For each sampling point $z$, we look for a solution to the far-field equation $F g=f_{z}$, where $F$ is the far-field operator and $f_{z}$ is a specific test-function. The solvability of the far-field equation is then used to determine the scatterer's shape. See [4] and references therein for a topical review. The Factorization Method [5, 6] is an alternative to retrieve exactly an obstacle's shape by solving the equation $|F|^{1 / 2} g=f_{z}$. In the case of absorbing inhomogeneous media, rather than obstacles, the Factorization Method has to be adapted. Inhomogeneous media are located by considering the operator $F_{\#}$ instead of $F$, where $F_{\#}=|\operatorname{Re} F|+|\operatorname{Im} F|[7,8]$. This family of methods allows to identify scatterers in the air by taking advantage of the very simple expression of the far field of the Green function. With (non-absorbing) inhomogeneous background media, it is only recently that a Factorization Method has been proposed to reconstruct the shape of obstacles [9]. We address the problem of reconstructing the support of a perturbation in an inhomogeneous background medium from farfield measurements. In the near field case for electromagnetic waves, this would be the electrical impedance tomography (EIT) $[10,11]$. Hence, in this paper, targeted applications should be non-destructive investigations $[12,13]$.

Consider the case where the acoustic refraction index of some inhomogeneous anisotropic medium is assumed to be known. It may happen that, in some places, the actual index is different from the reference value, as seen in Figure 1. This could happen for instance from a perturbation, or a deterioration of the actual index. So we say there is a defect at any point where 

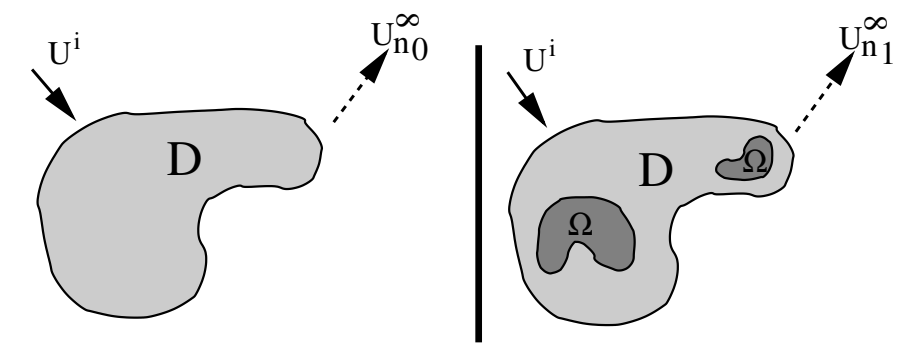

Figure 1: Reference setting (left) and actual setting (right).

the reference index is different from the actual index. Our main result is an explicit localization of the defects. This localization is obtained from the reference index and measurements gathered in the actual setting. To achieve this, we use those measurements to build an operator $\widetilde{W}_{\#}$ on which we can apply the Factorization Method. With $\left\{\sigma_{j}, \Psi_{j}\right\}$ being an eigensystem for $\widetilde{W}_{\#}$, we show that the support $\Omega$ of the defects is characterized by the relation

$$
z \in \Omega \Longleftrightarrow 0<w_{\left\{n_{0}, n_{1}\right\}}(z):=\left(\sum_{j} \frac{\left|\left\langle\overline{u_{n_{0}}(\cdot, z)}, \psi_{j}\right\rangle_{L^{2}\left(S^{d-1}\right)}\right|^{2}}{\sigma_{j}}\right)^{-1}
$$

where $u_{n_{0}}$ are near-field data computed from the reference index. This leads to a fast algorithm to reconstruct the shape of defects by plotting the values of $w_{\left\{n_{0}, n_{1}\right\}}$. Moreover, since our formulation does not rely on Green functions but on near-field data, the indicator function $w_{\left\{n_{0}, n_{1}\right\}}$ is easy to compute. Typically, the near-field data $u_{n_{0}}$ have already been computed in the process of building the matrix representation of the operator $\widetilde{W}_{\#}$.

In a first part of this paper, we will set the notations (section 2). Then, we develop the two main steps of this method. First, we characterize the location of the defects by a set of test functions (section 3). Then, these test functions are linked to the measurements by the Factorization Method (section 5). Thus, this step will involve a factorization of some measurement operator (section 4). Then, we can explicitly characterize the location of the defects from measurements (section 6). A second part will illustrate the numerical behaviour of this method in a large range of settings, including absorbing media and limited aperture data (section 7). We end by some conclusions.

\section{Presentation of the problem}

If we consider time-harmonic acoustic waves with a fixed wave number $k$, the spatial part of the wave equation is modeled by the Helmholtz equation. Inhomogeneous media are represented by an acoustic refraction index denoted by $n(x)$ and normalized to 1 in the air. Then, let us denote by $D$ the support of $(n(x)-1)$. The directions of measurements are taken on a subset of the unit sphere $S^{d-1}$, where $d$ is the problem's dimension $(d=2$ or 3$)$. We denote this set of measurement directions by $\Gamma_{m}$ and the set of incidence directions for plane wave sources by $\Gamma_{e}$ (see figure 2).

We denote by $u^{i} \in L_{l o c}^{2}\left(\mathbb{R}^{d}\right)$ an incoming wave satisfying (3) with $n=1$. The scattered field is denoted by $u^{s} \in L_{l o c}^{2}\left(\mathbb{R}^{d}\right)$ and is assumed to satisfy the Sommerfeld radiation condition

$$
\partial_{r} u^{s}=i k u^{s}+o\left(|x|^{-\frac{d-1}{2}}\right) .
$$




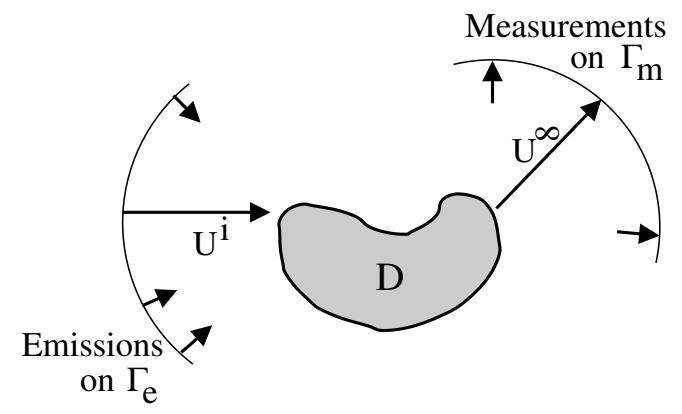

Figure 2: General setting and notations.

With a refraction index $n \in L^{\infty}\left(\mathbb{R}^{d}\right)$, the total field denoted by

$$
u_{n}:=u^{s}+u^{i}
$$

is assumed to satisfy the Helmholtz equation for inhomogeneous media

$$
\Delta u_{n}+k^{2} n(x) u_{n}=0, \quad x \in \mathbb{R}^{d} .
$$

The linear system (1)-(2)-(3) defines $u_{n}$ uniquely from $u^{i}$ and it is known to be invertible in $L^{2}(D)$. Thus, let us denote the corresponding automorphism by

$$
\begin{aligned}
\mathcal{T}_{n}: L^{2}(D) & \rightarrow L^{2}(D) \\
u^{i} & \mapsto u_{n}
\end{aligned}
$$

For practical reasons, we will mainly consider scattered waves having a plane-wave source defined by $u^{i}(\hat{x}, z)=\exp (i k z \cdot \hat{x})$, where $\hat{x} \in \Gamma_{e}$ is the incidence direction. Then, let us define the solution to (1)-(2)-(3) at the point $z \in \mathbb{R}^{d}$, with a plane-wave source of incoming direction $\hat{x}$, by

$$
u_{n}(\hat{x}, z):=\mathcal{T}_{n}\left(u^{i}(\hat{x}, \cdot)\right)(z) .
$$

Furthermore, the outgoing part of a wave has an asymptotic behaviour called the far field. Let $u_{n}^{\infty} \in L^{2}\left(\Gamma_{m}\right)$ denote the far field given by the Atkinson expansion [14]

$$
u_{n}(x)=u^{i}(x)+\gamma \frac{e^{i k|x|}}{|x|^{\frac{d-1}{2}}} u_{n}^{\infty}\left(\frac{x}{|x|}\right)+o\left(|x|^{-\frac{d-1}{2}}\right),
$$

where $\gamma$ depends only on the dimension and is defined by

$$
\gamma:= \begin{cases}\frac{e^{i \pi / 4}}{\sqrt{8 \pi k}} & \text { if } \mathrm{d}=2 \\ \frac{1}{4 \pi} & \text { if } \mathrm{d}=3\end{cases}
$$

We want to reconstruct the shape of defects in a reference medium whose index is denoted by $n_{0} \in L^{\infty}(D)$. Let then $n_{1} \in L^{\infty}(D)$ denote the actual index, altered by the presence of these defects. So denote the support of the difference between the two indices (see figure 1) by

$$
\Omega:=\operatorname{support}\left(n_{1}-n_{0}\right) .
$$

The goal is to reconstruct the domain $\Omega$ from the reference index $n_{0}$ and far-field measurements $u_{n_{1}}^{\infty}$. 


\section{Characterization of the domain $\Omega$ by test functions}

The characterization of the defects' location is based on an adaptation to our case of identification by point-sources. Thus, we denote the fundamental solution to system (1)-(2)-(3) by

$$
\Phi_{n}(z, x), \quad z, x \in \mathbb{R}^{d},
$$

and its far field by

$$
\Phi_{n}^{\infty}(z, \hat{x}), \quad z \in \mathbb{R}^{d}, \hat{x} \in S^{d-1} .
$$

With help of this fundamental solution we can give solutions by potentials. So let us denote the volumic potential corresponding to the refraction index $n$ (see [15, pages 158 and following]) by $V_{n}: L^{2}(D) \rightarrow \mathcal{C}^{0}\left(\mathbb{R}^{d}\right)$ and define it by

$$
V_{n} h(x)=\left\langle h, \overline{\Phi_{n}(\cdot, x)}\right\rangle_{L^{2}(D)},
$$

where $\langle\cdot, \cdot\rangle_{L^{2}(D)}$ stands for the usual hermitian inner product for the Hilbert space $L^{2}(D)$, that is

$$
\langle f, g\rangle_{L^{2}(D)}=\int_{D} f \bar{g}
$$

Furthermore, its asymptotic behaviour is given by the operator $V_{n}^{\infty}: L^{2}(D) \rightarrow L^{2}\left(\Gamma_{m}\right)$ defined by

$$
V_{n}^{\infty} h(\hat{x}):=\left(V_{n} h\right)^{\infty}(\hat{x})=\left\langle h, \overline{\Phi_{n}^{\infty}(\cdot, \hat{x})}\right\rangle_{L^{2}(D)} .
$$

We can now state a first characterization of $\Omega$ by test functions:

Proposition 3.1. For each $z \in \mathbb{R}^{3}$, we have

$$
z \in \Omega \Longleftrightarrow \Phi_{n_{0}}^{\infty}(z, \cdot) \in \mathcal{R}\left(V_{n_{0}}^{\infty} \chi_{\Omega}\right)
$$

Proof. Let us begin by building a pre-image of $\Phi_{n_{0}}^{\infty}(z, \cdot)$. Let $z \in \Omega$ and choose a ball $B_{z, \varepsilon}$, with center $z$ and radius $\varepsilon$, included in $\Omega$. Let then $f_{z}$ be a smooth function being equal to $\Phi_{n_{0}}^{\infty}(z, \cdot)$ out of $B_{z, \varepsilon}$ and to 0 in $B_{z, \varepsilon / 2}$. We denote by $\partial_{\nu}$ the normal derivative and by $\partial_{\Omega}$ the boundary of $\Omega$. Thus, we have $f_{z}=\Phi_{n_{0}}^{\infty}(z, \cdot)$ on $\partial_{\Omega}, \partial_{\nu} f_{z}=\partial_{\nu} \Phi_{n_{0}}^{\infty}(z, \cdot)$ on $\partial_{\Omega}$ and $\left(\Delta+k^{2} n_{0}\right) f_{z}=0$ out of $B_{z, \varepsilon}$. We will then write a representation formula for this function. Green's formula applied to $f_{z}$ and $\Phi_{n_{0}}^{\infty}(x, \cdot)$ leads to

$$
\begin{aligned}
\int_{\Omega}( & \left.f_{z} \Delta \Phi_{n_{0}}(x, \cdot)-\Phi_{n_{0}}(x, \cdot) \Delta f_{z}\right) \\
& =\int_{\partial \Omega}\left(f_{z} \partial_{\nu} \Phi_{n_{0}}(x, \cdot)-\Phi_{n_{0}}(x, \cdot) \partial_{\nu} f_{z}\right)
\end{aligned}
$$

Since $\Delta \Phi_{n_{0}}+k^{2} n_{0} \Phi_{n_{0}}=-\delta_{x}$ and $f_{z}=\Phi_{n_{0}}$ on $\partial_{\Omega}$, we have

$$
\begin{aligned}
\int_{\Omega}( & \left.f_{z}\left(-\delta_{x}-\left(k^{2} n_{0}\right) \Phi_{n_{0}}(x, \cdot)\right)-\Phi_{n_{0}}(x, \cdot) \Delta f_{z}\right) \\
& =\int_{\partial \Omega}\left(\Phi_{n_{0}}(z, \cdot) \partial_{\nu} \Phi_{n_{0}}(x, \cdot)-\Phi_{n_{0}}(x, \cdot) \partial_{\nu} \Phi_{n_{0}}(z, \cdot)\right) .
\end{aligned}
$$

Finally, by Green's formula, and recalling that $\Phi_{n_{0}}$ is a fundamental solution, we obtain

$$
-f_{z}(x)-\int_{\Omega}\left(\Delta+k^{2} n_{0}\right) f_{z} \Phi_{n_{0}}(x, \cdot)=\Phi_{n_{0}}(z, x)-\Phi_{n_{0}}(x, z) .
$$


By the symmetry of Green functions, this reduces to

$$
f_{z}(x)=-\int_{\Omega}\left(\Delta+k^{2} n_{0}\right) f_{z} \Phi_{n_{0}}(\cdot, x) .
$$

Since $f_{z}$ is equal to $\Phi_{n_{0}}$ out of $\Omega$, we can consider the asymptotic behaviour of the previous equation

$$
\Phi_{n_{0}}^{\infty}(z, \hat{x})=f_{z}^{\infty}(\hat{x})=-V_{n_{0}}^{\infty}\left[\chi_{\Omega}\left(\Delta+k^{2} n_{0}\right) f_{z}\right](\hat{x}),
$$

where $\chi_{\Omega}$ is the characteristic function of $\Omega$. This proves the implication.

Conversely, assume that $z \notin \Omega$ and that there is some function $f_{z}$ such that $\Phi_{n_{0}}^{\infty}(z, \hat{x})=$ $V_{n_{0}}^{\infty} f_{z}(\hat{x})$. By Rellich's lemma we have $\Phi_{n_{0}}(z, x)=V_{n_{0}} f_{z}(x)$ out of $\Omega \cup\{z\}$. However, the righthand side term is continuous at $z$, while the left-hand side is singular, which is not possible.

Rather than characterizing $\Omega$ with the help of fundamental solutions, we state a similar characterization, now relying on near-field data:

Theorem 3.2. Let us define the operator $C: L^{2}(D) \rightarrow L^{2}\left(\Gamma_{e}\right)$ by

$$
C f(\hat{x})=\left\langle f, u_{n_{0}}(\hat{x}, \cdot)\right\rangle_{L^{2}(D)} .
$$

For each $z \in \mathbb{R}^{d}$, we have

$$
z \in \Omega \Longleftrightarrow \overline{u_{n_{0}}(\cdot, z)} \in \mathcal{R}\left(C \chi_{\Omega}\right)
$$

Proof. We make use of the mixed reciprocity principle (see [9, equation (3.66)])

$$
\Phi_{n}^{\infty}(z, \hat{x})=u_{n}(-\hat{x}, z)
$$

to obtain Theorem 3.2 from Proposition 3.1. Indeed, from this proposition we have that $z \in \Omega$ if and only if there is a function $g_{z} \in L^{2}(\Omega)$ such that

$$
\Phi_{n_{0}}^{\infty}(z, \hat{x})=\left\langle g_{z}, \overline{\Phi_{n_{0}}^{\infty}(\cdot, \hat{x})}\right\rangle_{L^{2}(\Omega)}
$$

Using the relation (5), this means

$$
u_{n_{0}}(-\hat{x}, z)=\left\langle g_{z}, \overline{u_{n_{0}}(-\hat{x}, \cdot)}\right\rangle_{L^{2}(\Omega)}
$$

Thus, the function $f_{z}=\overline{\chi_{\Omega} g_{z}}$ satisfies $\overline{u_{n_{0}}(\cdot, z)}=C \chi_{\Omega} f_{z}$.

Remark 3.3. We have characterized $\Omega$ by using near-field solutions rather than fundamental solutions. A similar approach is followed in [16] where the test functions are derived from an adjoint problem. When the far field data $u_{n_{0}}^{\infty}$ is required, it is usually computed from the nearfield data $u_{n_{0}}$. Thus, the test functions involved in Theorem 3.2 will already be computed in any inverse problem relying on a given reference far-field.

\section{Factorization of the measurement operator}

We have exhibited two operators, both characterizing the domain $\Omega$ by their ranges, each of them through a specific set of test functions. Since the assumed reference index $n_{0}$ is known, the test function $u_{n_{0}}(\cdot, z)$ can be evaluated. However, the operator $C \chi_{\Omega}$ requires the explicit 
knowledge of $\Omega$ to be defined. Thus, we need to find a way to connect these test functions to the measurements.

We are looking for the location of defects. Thus, following [10], our measurement operator will be the difference between the classical far-field operators corresponding respectively to the actual index and the reference one. Denoting the classical far-field operator $F_{n}: L^{2}\left(\Gamma_{e}\right) \rightarrow L^{2}\left(\Gamma_{m}\right)$ by

$$
F_{n} g(\hat{x})=\left\langle g, \overline{u_{n}^{\infty}(\hat{x}, \cdot)}\right\rangle_{L^{2}\left(\Gamma_{e}\right)},
$$

we define the measurement operator by

$$
W^{\infty}:=F_{n_{1}}-F_{n_{0}} .
$$

To connect the ranges of $W^{\infty}$ and $C \chi_{\Omega}$, we will follow the Factorization Method. This method relies on some symmetric factorization $F=H^{\star} T H$ of the far-field operator $F$, where $H^{\star}$ characterizes the domain which has to be located. Thus, we need a factorization of $W^{\infty}$ involving the operator $C$ defined in Theorem 3.2.

This factorization comes from integral representations, also known as the Lippmann-Schwinger equations, for solutions to (1)-(2)-(3).

Lemma 4.1. Denoting the subtraction between the total fields generated by the reference index and the actual one by

$$
w=u_{n_{1}}-u_{n_{0}},
$$

and by $I$ the identity operator, we have the following integral representations in $L^{2}(D)$ :

$$
\begin{aligned}
u_{n_{0}}= & \left(I+V_{n_{0}} k^{2}\left(n_{0}-1\right)\right) u^{i}, \\
u_{n_{1}}= & \left(I-V_{n_{0}} k^{2}\left(n_{1}-n_{0}\right)\right)^{-1}\left(I+V_{n_{0}} k^{2}\left(n_{0}-1\right)\right) u^{i}, \\
w= & V_{n_{0}} k^{2}\left(n_{1}-n_{0}\right) \\
& \quad\left(I-V_{n_{0}} k^{2}\left(n_{1}-n_{0}\right)\right)^{-1}\left(I+V_{n_{0}} k^{2}\left(n_{0}-1\right)\right) u^{i} .
\end{aligned}
$$

These representations rely on the uniqueness of solutions, which is a consequence of the unique continuation principle.

Lemma 4.2 (Unique Continuation Principle). [17, Theorem 2.1.4] Let $n \in L^{\infty}\left(\mathbb{R}^{d}\right)$ satisfy $n(x)=1$ for $|x| \geqslant a$. Let $u_{n} \in L_{\text {loc }}^{2}\left(\mathbb{R}^{d}\right)$ denote a solution to (3) in $\mathbb{R}^{d}$ such that $u_{n}(x)=0$ for $|x| \geqslant b$, were $b$ is some constant such that $b \geqslant a$. Then, we have $u_{n}(x)=0$ on $\mathbb{R}^{d}$.

Proof of Lemma 4.1. We will use the fundamental solution $\Phi_{n_{0}}(z, \cdot)$ to write the integral representations (7) and (8). The integral representation (9) will then follow by subtraction.

Let $z \in \mathbb{R}^{d}$. First, we isolate in (3) the term $\left(u_{n}-u^{i}\right)$, which satisfies the same Sommerfeld radiation condition as $\Phi_{n_{0}}(z, \cdot)$

$$
\begin{aligned}
\left(\Delta+k^{2} n\right) u_{n}=(\Delta+ & \left.k^{2} n_{0}\right)\left(u_{n}-u^{i}\right) \\
& +k^{2}\left(n_{0}-1\right) u^{i}+k^{2}\left(n-n_{0}\right) u_{n} .
\end{aligned}
$$

Thus, we can apply Green's formula to obtain

$$
\begin{aligned}
\int_{D} \Phi_{n_{0}}(z, \cdot)\left(\Delta+k^{2} n_{0}\right)\left(u_{n}-u^{i}\right) \\
=\int_{D}\left(u_{n}-u^{i}\right)\left(-\left(k^{2} n_{0}\right) \Phi_{n_{0}}(z, \cdot)-\delta_{z}\right) \\
\quad \quad+\int_{D} \Phi_{n_{0}}(z, \cdot)\left(k^{2}+k^{2}\left(n_{0}-1\right)\right)\left(u_{n}-u^{i}\right) \\
\quad=-\left(u_{n}-u^{i}\right)(z) .
\end{aligned}
$$


So, multiplying (3) by $\Phi_{n_{0}}(z, \cdot)$ and integrating over $D$, it yields

$$
-\left(u_{n}-u^{i}\right)+V_{n_{0}} k^{2}\left(n_{0}-1\right) u^{i}+V_{n_{0}} k^{2}\left(n-n_{0}\right) u_{n}=0 .
$$

By setting successively $n$ equal to $n_{0}$ and $n_{1}$, we obtain

$$
\begin{aligned}
& u_{n_{0}}=u^{i}+V_{n_{0}} k^{2}\left(n_{0}-1\right) u^{i}, \\
& u_{n_{1}}-V_{n_{0}} k^{2}\left(n_{1}-n_{0}\right) u_{n_{1}}=u^{i}+V_{n_{0}} k^{2}\left(n_{0}-1\right) u^{i} .
\end{aligned}
$$

Then, the Fredholm alternative and the unique continuation principle give us the invertibility of the operator $\left(I-V_{n_{0}} k^{2}\left(n_{1}-n_{0}\right)\right)$. This yields the representations (7) and (8).

Corollary 4.3. The product $\mathcal{T}_{n_{1}} \mathcal{T}_{n_{0}}^{-1}$ has the following integral representation:

$$
\mathcal{T}_{n_{1}} \mathcal{T}_{n_{0}}^{-1}=\left(I-V_{n_{0}} k^{2}\left(n_{1}-n_{0}\right)\right)^{-1} .
$$

It is furthermore an automorphism in $L^{2}(\mathcal{O})$ for any open open set $\mathcal{O}$ containing $\Omega$ and it maps functions satisfying (3) with $n=n_{0}$ into functions satisfying (3) with $n=n_{1}$.

Moreover, we have

$$
\chi_{\Omega} \mathcal{T}_{n_{1}} \mathcal{T}_{n_{0}}^{-1}=\chi_{\Omega} \mathcal{T}_{n_{1}} \mathcal{T}_{n_{0}}^{-1} \chi_{\Omega}
$$

Proof. Recognizing the integral representation for $u_{n_{0}}$ in (7) as the last term of the integral representation for $u_{n_{1}}$ in (8), it yields $\mathcal{T}_{n_{1}}=\left(I-V_{n_{0}} k^{2}\left(n_{1}-n_{0}\right)\right)^{-1} \mathcal{T}_{n_{0}}$.

Furthermore, this shows that $\left(I-V_{n_{0}} k^{2}\left(n_{1}-n_{0}\right)\right)^{-1}$ maps total fields for the index $n_{0}$ into total fields for the index $n_{1}$.

We conclude that $\left(I-V_{n_{0}} k^{2}\left(n_{1}-n_{0}\right)\right)$ is an automorphism in $L^{2}(\mathcal{O})$ for any open set $\mathcal{O}$ containing $\Omega$ from the Fredholm alternative by using the unique continuation principle.

Finally, any function $h$ satisfying $\left.h\right|_{\Omega}=0$ also satisfies $\left(I-V_{n_{0}} k^{2}\left(n_{1}-n_{0}\right)\right) h=h$, and thus $\chi_{\Omega} \mathcal{T}_{n_{1}} \mathcal{T}_{n_{0}}^{-1}=\chi_{\Omega} \mathcal{T}_{n_{1}} \mathcal{T}_{n_{0}}^{-1} \chi_{\Omega}$

Corollary 4.4. The subtraction $w$ can be obtained as image of the source $u^{i}$ by the following product of operators:

$$
w=V_{n_{0}} k^{2}\left(n_{1}-n_{0}\right) \chi_{\Omega} \mathcal{T}_{n_{1}} \mathcal{T}_{n_{0}}^{-1} \chi_{\Omega} \mathcal{T}_{n_{0}} u^{i} .
$$

Proof. This follows immediately from (9) and Corollary 4.3.

Now that we have a characterization of $w$ as image of a source $u^{i}$ by a product of operators, we obtain a factorization for $W^{\infty}$.

Lemma 4.5. Defining the operator $A$ by

$$
A:=k^{2}\left(n_{1}-n_{0}\right) \mathcal{T}_{n_{1}} \mathcal{T}_{n_{0}}^{-1},
$$

the measurement operator $W^{\infty}$ has a factorization of the form

$$
W^{\infty}=V_{n_{0}}^{\infty} A C^{\star} .
$$


Proof. Let us define the operator $H: L^{2}\left(\Gamma_{e}\right) \rightarrow L^{2}(D)$ by

$$
H g(z)=\left\langle g, \Phi_{1}^{\infty}(z, \cdot)\right\rangle_{L^{2}\left(\Gamma_{m}\right)} .
$$

Since $\Phi_{1}^{\infty}(z, \hat{x})=e^{i k z \cdot \hat{x}}$, this is a superposition of plane waves and $H g$ is the Herglotz wave function with kernel $g$. Thus, by (6), $W^{\infty} g$ is the far field of $\left(\mathcal{T}_{n_{1}}-\mathcal{T}_{n_{1}}\right) H g$ and it follows from Corollary 4.4 that $W^{\infty}=V_{n_{0}}^{\infty} k^{2}\left(n_{1}-n_{0}\right) \mathcal{T}_{n_{1}} \mathcal{T}_{n_{0}}^{-1} \mathcal{T}_{n_{0}} H$. It is easy to see that

$$
C^{\star}=\mathcal{T}_{n_{0}} H,
$$

and thus, replacing $k^{2}\left(n_{1}-n_{0}\right) \mathcal{T}_{n_{1}} \mathcal{T}_{n_{0}}^{-1}$ by $A$ achieves the proof.

Our factorization of $W^{\infty}$ involves $V_{n_{0}}^{\infty}$ and $C$, which both have been shown to characterize $\Omega$. But this does not lead to a reconstruction algorithm. If we had a symmetric factorization, the Factorization method would provide such an algorithm. Thus, we need a (one-to-one) relation between the operators $V_{n_{0}}^{\infty}$ and $C$. In the proof of Theorem 3.2, and more specifically in relation (5), we have already seen that these operators differ by a complex conjugate and a symmetry with respect to the direction $\hat{x}$. This is the principle of time reversal, which maps an outgoing wave onto an incoming wave [18]. The operator linking the far field of outgoing waves to the far field of incoming waves is called the scattering operator [19, chapter X, §3]. Thus, we will use the scattering operator to $\operatorname{link} V_{n_{0}}^{\infty}$ and $C$. So let us define the scattering operator $S_{n}: L^{2}\left(S^{d-1}\right) \rightarrow L^{2}\left(S^{d-1}\right)$ by

$$
S_{n}:=I+2 i k|\gamma|^{2} F_{n} .
$$

We then have the following relation.

Lemma 4.6. Assume that the refraction indices are real-valued $\left(n_{0}, n_{1} \in \mathbb{R}\right)$ and that measurements as well as incidence directions are spread over the whole unit sphere $\left(\Gamma_{m}=\Gamma_{e}=S^{d-1}\right)$. Then, the operators $V_{n_{0}}^{\infty}$ and $C$ satisfy

$$
V_{n_{0}}^{\infty}=S_{n_{0}} C
$$

Proof. Let $\theta \in \Gamma_{e}$. The operator $C$, as defined is Theorem 3.2, is the scalar product by $u_{n_{0}}(\theta, \cdot)$, which can also be written as $\mathcal{T}_{n_{0}}\left[\overline{\Phi_{1}(\cdot, \theta)}\right]$. Relation (5) shows that the operator $V_{n_{0}}^{\infty}$, as defined by (4), is the scalar product by $\overline{\mathcal{T}_{n_{0}}\left[\Phi_{1}(\cdot, \theta)\right]}$. Recalling the integral representation (7), we deduce a representation of the subtraction of (the complex conjugate of) those functions:

$$
\begin{aligned}
\mathcal{T}_{n_{0}} & {\left[\Phi_{1}^{\infty}(\cdot, \theta)\right](z)-\overline{\mathcal{T}_{n_{0}}\left[\overline{\Phi_{1}^{\infty}(\cdot, \theta)}\right](z)} } \\
& =\int_{y \in \mathbb{R}^{d}}\left(\Phi_{n_{0}}(y, z)-\overline{\Phi_{n_{0}}(y, z)}\right) k^{2}\left(n_{0}(y)-1\right) \Phi_{1}^{\infty}(y, \theta) .
\end{aligned}
$$

Hence, we have to evaluate the imaginary part of the fundamental solution. For any $y, z \in \mathbb{R}^{d}$ and $R$ such that the ball $B_{R}$ of radius $R$ contains $y$ and $z$, we have

$$
\begin{aligned}
\Phi_{n_{0}}(y, z) & -\overline{\Phi_{n_{0}}(y, z)} \\
=- & \int_{x \in B_{R}} \Phi_{n_{0}}(y, x)\left(\Delta+k^{2} n_{0}(x)\right) \overline{\Phi_{n_{0}}(z, x)} \\
& \quad+\int_{x \in B_{R}} \overline{\Phi_{n_{0}}(z, x)}\left(\Delta+k^{2} n_{0}(x)\right) \Phi_{n_{0}}(y, x) \\
=- & \int_{x \in S_{R}}\left(\Phi_{n_{0}}(y, x) \partial_{\nu} \overline{\Phi_{n_{0}}(z, x)}-\overline{\Phi_{n_{0}}(z, x)} \partial_{\nu} \Phi_{n_{0}}(y, x)\right) .
\end{aligned}
$$


Since $\Phi_{n_{0}}(z, x)$ is outgoing, and thus has a far field, letting $R$ go to infinity in the last equation yields

$$
\Phi_{n_{0}}(y, z)-\overline{\Phi_{n_{0}}(y, z)}=2 i k|\gamma|^{2} \int_{\hat{x} \in S^{d-1}} \Phi_{n_{0}}^{\infty}(y, \hat{x}) \overline{\Phi_{n_{0}}^{\infty}(z, \hat{x})}
$$

Hence, by recalling that $\Phi_{1}^{\infty}(\cdot, \theta)=u^{i}(-\theta, \cdot)$ and using the reciprocity principle, it holds that

$$
\begin{aligned}
\mathcal{T}_{n_{0}}\left[\Phi_{1}^{\infty}(\cdot, \theta)\right](z)-\overline{\mathcal{T}_{n_{0}}\left[\overline{\Phi_{1}^{\infty}(\cdot, \theta)}\right](z)} \\
=\int_{\substack{y \in \mathbb{R}^{d}, \hat{x} \in S^{d-1}}} 2 i k|\gamma|^{2} \Phi_{n_{0}}^{\infty}(y, \hat{x}) \overline{\Phi_{n_{0}}^{\infty}(z, \hat{x})} k^{2}\left(n_{0}(y)-1\right) \Phi_{1}^{\infty}(y, \theta) \\
=\int_{\hat{x} \in S^{d-1}} 2 i k|\gamma|^{2} \overline{\Phi_{n_{0}}^{\infty}(z, \hat{x})} V_{n_{0}}^{\infty}\left[k^{2}\left(n_{0}-1\right) \Phi_{1}^{\infty}(\cdot, \theta)\right](\hat{x}) \\
=\int_{\hat{x} \in S^{d-1}} 2 i k|\gamma|^{2} \overline{\Phi_{n_{0}}^{\infty}(z, \hat{x})} V_{n_{0}}^{\infty}\left[k^{2}\left(n_{0}-1\right) \Phi_{1}^{\infty}(\cdot, \hat{x})\right](\theta) \\
=2 i k|\gamma|^{2} V_{n_{0}}^{\infty} k^{2}\left(n_{0}-1\right) H\left[\overline{\Phi_{n_{0}}^{\infty}(z,-\cdot)}\right](\theta) \\
=2 i k|\gamma|^{2} F_{n_{0}}\left[\overline{\left.\mathcal{T}_{n_{0}}\left[\overline{\Phi_{1}^{\infty}}\right](z)\right]}(\theta),\right.
\end{aligned}
$$

where $\Phi_{n_{0}}^{\infty}(z,-\cdot): \hat{x} \mapsto \Phi_{n_{0}}^{\infty}(z,-\hat{x})$ is the function $\Phi_{n_{0}}^{\infty}(z, \cdot)$ with the change of variables $\hat{x} \mapsto-\hat{x}$, which is needed to obtain the expression of the operator $H$. Finally, this reduces to $\mathcal{T}_{n_{0}}\left[\Phi_{1}^{\infty}(\cdot, \theta)\right](z)=\left(I+2 i k|\gamma|^{2} F_{n_{0}}\right) \overline{\mathcal{T}_{n_{0}}\left[\overline{\Phi_{1}^{\infty}}\right](z)}$ and ends the proof.

We are now able to build an operator from the physical measurements which has a symmetric factorization.

Corollary 4.7. Under the assumptions of Lemma 4.6, the operator $\widetilde{W}: L^{2}\left(S^{d-1}\right) \rightarrow L^{2}\left(S^{d-1}\right)$ defined by

$$
\widetilde{W}:=S_{n_{0}}^{\star} W^{\infty}
$$

has a factorization of the form

$$
\widetilde{W}=C A C^{\star}
$$

Proof. Lemma 4.5, yields the factorization $W^{\infty}=V_{n_{0}}^{\infty} A C^{\star}$. From Lemma 4.6 we see that $V_{n_{0}}^{\infty}=S_{n_{0}} C$. Thus, $W^{\infty}=S_{n_{0}} C A C^{\star}$. Since $S_{n_{0}}$ is unitary, the result is straightforward.

Now we have a symmetric factorization holding for an operator $\widetilde{W}$ built upon the physical measurements. This factorization clearly involves the operator $C$ which has been used in Theorem 3.2 to characterize $\Omega$ by its range. Thus, the next step is to show a relation between the ranges of $C$ and $\widetilde{W}$.

\section{Linking the test functions to the measurements}

Theorem 3.2 provides test functions characterizing $\Omega$ through the range of $C \chi_{\Omega}$. The operator $C \chi_{\Omega}$ is not available through measurements but $\widetilde{W}$ is. Furthermore, Corollary 4.7 gives the relation $\widetilde{W}=C A C^{\star}$. The Factorization method is a way of linking ranges of operators. The initial version states that if some operator $F$ is normal and has a factorization of the form $H T H^{\star}$, under some additional assumptions on $H$ and $T$, the ranges of $|F|^{1 / 2}$ and $H$ coincide. Under the assumptions of Lemma 4.6 , it is known that the far-field operator $F_{n}$ is normal [8, Theorem 4.4]. 
But the set of normal operators is not a group, so the operator $\widetilde{W}$ has no reason to be normal. Thus, we will use a second version of the factorization method for non-normal operators:

Proposition 5.1 (The $F_{\#}$ Method). [20, Theorem 2.1] Let $X \subset U \subset X^{\star}$ be a Gelfand triple, where $U$ is a Hilbert space and $X$ a reflexive Banach space such that the embeddings are dense. Furthermore, let $Y$ be a second Hilbert space and let $F: Y \rightarrow Y, H: X \rightarrow Y$ and $T: X^{\star} \rightarrow X$ be bounded linear operators such that

$$
F=H T H^{\star} \text {. }
$$

We make the following assumptions:

1. $H$ is compact and has dense range,

2. $\operatorname{Re} T$ has the form $\operatorname{Re} T=G+K$ with some compact operator $K$ and some self-adjoint coercive operator $G: X^{\star} \rightarrow X$,

3. $\operatorname{Im} T$ is non-negative on $\mathcal{R}\left(G^{\star}\right)$,

4. $T$ is one-to-one, or $\operatorname{Im} T$ is strictly positive on $\operatorname{ker}(\operatorname{Re} T) \backslash\{0\}$.

Then, the operator $F_{\#}=|\operatorname{Re} F|+\operatorname{Im} F$ is positive and the ranges of $H$ and $F_{\#}^{\frac{1}{2}}$ coincide.

Since we have a symmetric factorization for $\widetilde{W}$, we will use Proposition 5.1 to link the ranges of $\widetilde{W}_{\#}$ and $C \chi_{\Omega}$. The assumptions of this theorem require some coercivity. As we will see, this is related to the contrast between the reference index $n_{0}$ and the actual values of $n_{1}$, i.e. the defects should be clearly distinguished from the background. Thus, we will make the following geometrical assumption.

Assumption 5.2. Assume that $n_{0}$ and $n_{1}$ are real valued and that either $\left(n_{1}-n_{0}\right)$ or $\left(n_{0}-n_{1}\right)$ is locally bounded from below :

- for any compact subset $\omega$ included in $\Omega$, there exists $c>0$ such that $\left(n_{1}(z)-n_{0}(z)\right) \geqslant c$ for almost all $z \in \omega$,

or

- for any compact subset $\omega$ included in $\Omega$, there exists $c>0$ such that $\left(n_{0}(z)-n_{1}(z)\right) \geqslant c$ for almost all $z \in \omega$.

To use Proposition 5.1, we have to show that the operator $A$ defined in Lemma 4.5 satisfies the assumptions (2),(3) and (4).

Lemma 5.3. The operator $A$, defined in Lemma 4.5, satisfies $\operatorname{Im}\langle A \varphi, \varphi\rangle \geqslant 0, \forall \varphi \in \overline{\mathcal{R}\left(C^{\star}\right)}$. Furthermore, under Assumption 5.2 we have $A=A_{0}+K$, where $A_{0}$ is a self-adjoint coercive operator and $K$ is a compact operator.

Proof. Choose $\varphi \in \mathcal{R}\left(C^{\star}\right)$. This is a total field for the refraction index $n_{0}$. Hence, there exists an incident field $f$ such that $\varphi=\mathcal{T}_{n_{0}}(f)$. Let us set $u_{n_{0}}=\varphi$ and $u_{n_{1}}=\mathcal{T}_{n_{1}}(f)$. Thus, we obtain $A \varphi=k^{2}\left(n_{1}-n_{0}\right) u_{n_{1}}$. Moreover, choosing $R$ such that the ball $B_{R}$ of radius $R$ contains $\Omega$, it 
holds that

$$
\begin{aligned}
& \int_{\Omega} k^{2}\left(n_{1}-n_{0}\right) u_{n_{1}}\left(\overline{u_{n_{0}}-u_{n_{1}}}\right) \\
& =\int_{B_{R}}\left(\Delta+k^{2} n_{0}\right)\left(u_{n_{1}}-u_{n_{0}}\right)\left(\overline{u_{n_{1}}-u_{n_{0}}}\right) \\
& =\int_{B_{R}} k^{2} n_{0}\left|u_{n_{1}}-u_{n_{0}}\right|^{2}-\left|\nabla\left(u_{n_{1}}-u_{n_{0}}\right)\right|^{2} \\
& \quad+\int_{S_{R}}\left(\overline{u_{n_{1}}-u_{n_{0}}}\right) \partial_{\nu}\left(u_{n_{1}}-u_{n_{0}}\right)
\end{aligned}
$$

By letting $R$ go to infinity, it comes

$$
\begin{aligned}
& \int_{\Omega} k^{2}\left(n_{1}-n_{0}\right) u_{n_{1}}\left(\overline{u_{n_{0}}-u_{n_{1}}}\right) \\
& =\int_{R^{n}} k^{2} n_{0}\left|u_{n_{1}}-u_{n_{0}}\right|^{2}-\left|\nabla\left(u_{n_{1}}-u_{n_{0}}\right)\right|^{2} \\
& \quad+i k|\gamma|^{2} \int_{S^{d-1}}\left|u_{n_{1}}^{\infty}-u_{n_{0}}^{\infty}\right|^{2} .
\end{aligned}
$$

Hence, taking the imaginary part yields

$$
\operatorname{Im} \int_{\Omega} k^{2}\left(n_{1}-n_{0}\right) u_{n_{1}} \overline{u_{n_{0}}}=k|\gamma|^{2} \int_{S^{d-1}}\left|u_{n_{1}}^{\infty}-u_{n_{0}}^{\infty}\right|^{2} .
$$

This shows that $\operatorname{Im}\langle A \varphi, \varphi\rangle \geqslant 0$.

Furthermore, we also have that

$$
\begin{aligned}
\langle A \varphi, \varphi\rangle & =\int_{\Omega} k^{2}\left(n_{1}-n_{0}\right)\left|u_{n_{0}}\right|^{2}+\int_{\Omega} k^{2}\left(n_{1}-n_{0}\right)\left(u_{n_{1}}-u_{n_{0}}\right) \overline{u_{n_{0}}} \\
& =\left\langle A_{0} \varphi, \varphi\right\rangle+\langle K \varphi, \varphi\rangle,
\end{aligned}
$$

with $A_{0}=k^{2}\left(n_{1}-n_{0}\right) I$ and $K=k^{2}\left(n_{1}-n_{0}\right)\left(\mathcal{T}_{n_{1}} \mathcal{T}_{n_{0}}^{-1}-I\right)$. With Assumption 5.2, $A_{0}$ is clearly coercive and self-adjoint. Moreover, $\left(\mathcal{T}_{n_{1}} \mathcal{T}_{n_{0}}^{-1}-I\right)=\mathcal{T}_{n_{1}} \mathcal{T}_{n_{0}}^{-1}\left(I-\mathcal{T}_{n_{0}} \mathcal{T}_{n_{1}}^{-1}\right)$. By Corollary 4.3, it holds that $\mathcal{T}_{n_{0}} \mathcal{T}_{n_{1}}^{-1}=I-V_{n_{0}}\left(n_{1}-n_{0}\right)$. Thus, $K=k^{2}\left(n_{1}-n_{0}\right) \mathcal{T}_{n_{1}} \mathcal{T}_{n_{0}}^{-1} V_{n_{0}}\left(n_{1}-n_{0}\right)$. Since $V_{n_{0}}$ is compact and the other operators are bounded, $K$ is compact too. All these results are then extended to $\overline{\mathcal{R}\left(C^{\star}\right)}$ by continuity.

We have now met all the requirements to state the following result.

Proposition 5.4. With Assumption 5.2 and $\Gamma_{m}=\Gamma_{e}=S^{d-1}$, we have

$$
\mathcal{R}\left(\widetilde{W}_{\#}^{\frac{1}{2}}\right)=\mathcal{R}\left(C \chi_{\Omega}\right),
$$

where $\widetilde{W}_{\#}:=|\operatorname{Re} \widetilde{W}|+|\operatorname{Im} \widetilde{W}|$, with the convention $\operatorname{Re} \widetilde{W}=\frac{1}{2}\left(\widetilde{W}+\widetilde{W}^{\star}\right)$ and $\operatorname{Im} \widetilde{W}=\frac{1}{2 i} \widetilde{W}-$ $\left.\widetilde{W}^{\star}\right)$.

Proof. We apply Proposition 5.1 with $F=\widetilde{W}, H=C \chi_{\Omega}$ and $T=A$. Corollary 4.7 shows that the operator $W$ has a factorization of the form $C A C^{\star}$, where $A$ is an automorphism in $L^{2}(\Omega)$, as proved in Corollary 4.3. Thus, we obtain $\widetilde{W}=C \chi_{\Omega} A \chi_{\Omega} C^{\star}$ and satisfy assumption (4). The 
Herglotz wave operator $H$ is known to be injective and compact [21]. Thus, relation (11) shows that $C^{\star}$ is injective too. So, $C$ is compact and has dense range, which is assumption (1). Finally, assumptions (2) and (3) of Proposition 5.1 follow from Lemma 5.3. Indeed, if $A_{0}$ is self-adjoint coercive, so is $A_{0}+A_{0}^{\star}$ and if $K$ is compact, so is $K+K^{\star}$.

\section{Characterization of the domain $\Omega$ by measurements}

We have established that the domain $\Omega$ is characterized by the range of the operator $C \chi_{\Omega}$ and that this range coincides with the range of the operator $\widetilde{W}_{\#}^{\frac{1}{2}}$. Thus, we obtain a characterization of $\Omega$ by the range of $\widetilde{W}_{\#}^{\frac{1}{2}}$.

Theorem 6.1. Under Assumption 5.2 and with $\Gamma_{m}=\Gamma_{e}=S^{d-1}$ then, for each $z \in \mathbb{R}^{d}$, we have

$$
z \in \Omega \Longleftrightarrow w_{\left\{n_{0}, n_{1}\right\}}(z):=\left(\sum_{j} \frac{\left|\left\langle\overline{u_{n_{0}}(\cdot, z)}, \psi_{j}\right\rangle_{L^{2}\left(S^{d-1}\right)}\right|^{2}}{\sigma_{j}}\right)^{-1}>0,
$$

where $\sigma_{j} \in \mathbb{R}_{+}$are the eigenvalues of the positive self-adjoint operator $\widetilde{W}_{\#}$ and $\psi_{j} \in L^{2}\left(S^{d-1}\right)$ are the corresponding eigenfunctions.

Proof. We combine Theorem 3.2 and Proposition 5.4 to obtain

$$
z \in \Omega \Longleftrightarrow \overline{u_{n_{0}}(\cdot, z)} \in \mathcal{R}\left(\widetilde{W}_{\#}^{\frac{1}{2}}\right)
$$

Finally, the characterization of $\Omega$ by the function $w_{\left\{n_{0}, n_{1}\right\}}$ is Picard's range test criterion [22, Theorem A.51].

Thus, to obtain a visualization of the domain $\Omega$, we only have to plot the values of the function $w_{\left\{n_{0}, n_{1}\right\}}$ on a set of sampling points denoted by $\left\{z_{i}\right\}$.

Remark 6.2. The " $F_{\#}^{\frac{1}{2}}$ " method we used to locate defects is very close to the original " $\left(F^{\star} F\right)^{\frac{1}{4}}$ " method $[7,5]$. In both cases, a measurement operator $F$ (or $\left.F_{\#}\right)$ is built to have two factorizations. One of the form $H T H^{\star}$, with some operator $H$ characterizing the domain which has to be located, and another one of the form $F=|F|^{\frac{1}{2}} U|F|^{\frac{1}{2}}$. If the involved operators $T$ and $U$ are coercive, the ranges of $H$ and $|F|^{\frac{1}{2}}$ are proved to match [8, Theorem 1.21]. This allows to characterize the domain using the eigenvalues of an operator built from the measurements as we have done in Theorem 6.1.

However, a singular values decomposition (SVD) seems to unify both approaches. Indeed, it only requires the measurement operator to be compact [23]. This property is very stable and satisfied by every measurement operator studied so far. Furthermore, singular values are positive by nature. So, by considering singular values and the associated right singular functions instead of the eigenpairs $\left(\sigma_{j}, \Psi_{j}\right)$, the function $w_{\left\{n_{0}, n_{1}\right\}}$ is still well defined. By considering right singular functions, this is then the range test criterion for the operator $|F|^{\frac{1}{2}}$. Moreover, we have the natural factorization $|F|=|F|^{\frac{1}{2}}|F|^{\frac{1}{2}}$. This does neither require the indices to be real-valued nor even require the set of measurements directions to match the set of incidence directions: The SVD for matrices is well defined for rectangular matrices and we can apply it in our numerical implementations of the method. 
The whole difficulty is then transferred to the problem of showing the existence of some coercive operator $E$ such that $|F|=H E H^{\star}$. Indeed, let us suppose that there exists some coercive operator $E$ such that $\left|W^{\infty}\right|=C E C^{\star}$. We would then be able to prove that the ranges of the operators $\left|W^{\infty}\right|^{\frac{1}{2}}$ and $C \chi_{\Omega}$ coincide. Thus, we could characterize the support $\Omega$ of the defects by a function $w_{\left\{n_{0}, n_{1}\right\}}$ as defined in Theorem 6.1 , but where the set $\left\{\sigma_{j}, \Psi_{j}\right\}$ will be a right singular system for the measurement operator $W^{\infty}$ (instead of an eigensystem for $\left.\widetilde{W}_{\#}\right)$. We would then have a " $F^{\star} F^{\frac{1}{4}}$ "-like method, very simple to implement and which would cover complex-valued indices as well as limited aperture measurements with non-corresponding incident directions. That is, $\Gamma_{m}$ could be different from $S^{d-1}$, but also different from $\Gamma_{e}$. All our numerical experimentation validate this possibility.

\section{Numerical results}

In this section, we study the behaviour of our method through several numerical examples. In all the simulations, a uniform random noise (given in $\%$ of the measurements) will be added to the values of the effective far-field measurements $\left(u_{n_{1}}^{\infty}\right)$. Data are generated through a $P^{1}$ finite elements discretization considering the geometry shown in Figure 1. The problem over $\mathbb{R}^{2}$ is reduced to a bounded computing domain with help of cartesian Perfectly Matched Layers (PML) [24]. From this, we form the matrices $\mathbf{F}_{n_{j}}(l, m)=u_{n_{j}}^{\infty}\left(\hat{x}_{l}, \hat{x}_{m}\right), j=0,1$. Due to the reciprocity principle, the product of operators $R F_{n_{0}} R$, involved in the scattering operator, is discretized by the matrix $\mathbf{F}_{n_{0}}^{T}$ (the transpose of $\mathbf{F}_{n_{0}}$ ). We then build a discrete version of $\widetilde{W}_{\#}$. Finally, we choose a set of sampling points $\left\{z_{i}\right\}_{i=1,2, \ldots}$ to be tested, interpolate the values of $u_{n_{0}}\left(\hat{x}, z_{i}\right)$ and plot the values of $w_{\left\{n_{0}, n_{1}\right\}}\left(z_{i}\right)$. According to Theorem $6.1, w_{\left\{n_{0}, n_{1}\right\}}\left(z_{i}\right)$ should be near to 0 out of the defects and have higher values inside.

\subsection{Validation of the method}

First, the reference background index $n_{0}$ takes values in $[2.31,2.40]$ inside $D$. The actual index $n_{1}$ is then set equal to $n_{0}$ out of $\Omega$ and takes values in $[1.75,1.79]$ inside $\Omega$. The wave number $k$ is taken equal to 6 and the measurements are computed for 21 measurement/incidence directions evenly distributed over the unit circle.

Figure 3 presents the results obtained by plotting the values of $w_{\left\{n_{0}, n_{1}\right\}}$ with varying amounts of additional noise.

We notice that even with $10 \%$ additional noise, as shown in Figure 3b, both connected components of the defects are clearly reconstructed. With $30 \%$ additional noise, figure $3 \mathrm{c}$ shows strong perturbations but both defects are still correctly located. It is not until $50 \%$ additional noise, as presented in Figure 3d that the smallest defect does no longer stand out from the background. The left hand side defect being bigger, it is still visible but also begins to fade in the background noise and its shape is no longer correctly reconstructed.

Figure 4 presents the results obtained in the same conditions but with different wave numbers and with noise fixed at $2 \%$.

We notice that with a small wave number $(k=0.3)$, the defects are perceived as a single blurry area in Figure $4 \mathrm{a}$. In figure $4 \mathrm{~b}$, with $k=1$, the defects are still grouped under a single area, but this time it looks more like a convex hull. When $k=3$, both defects are clearly isolated and if we set the wave number even higher we notice a sharpening of the picture, as seen on figures $4 \mathrm{c}$ 


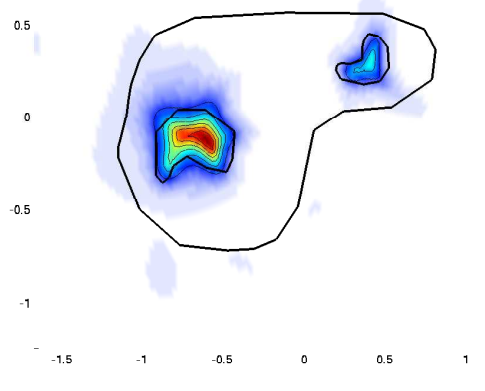

(a) $2 \%$ noise

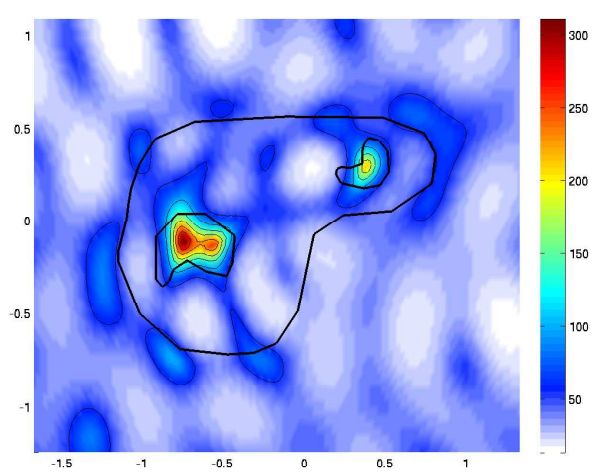

(c) $30 \%$ noise

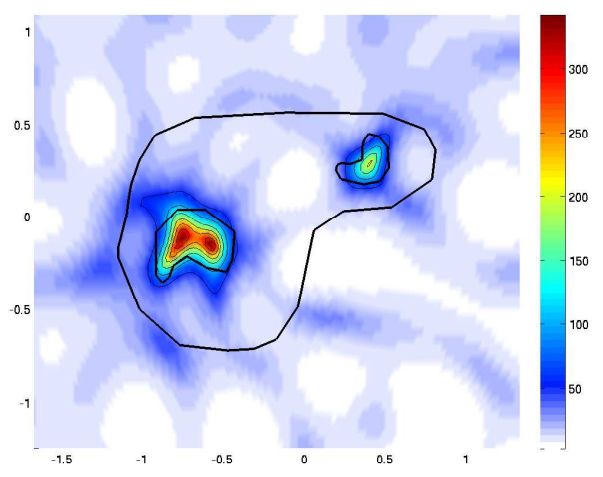

(b) $10 \%$ noise

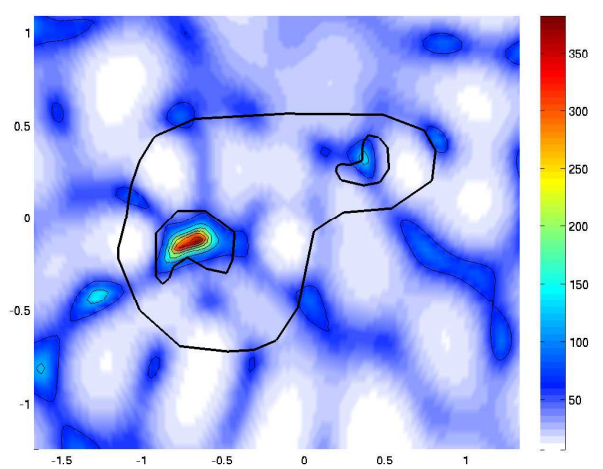

(d) $50 \%$ noise

Figure 3: Values of $w_{\left\{n_{0}, n_{1}\right\}}$ for real-valued indices with varying amounts of noise added to the measurements of the actual refraction index. 


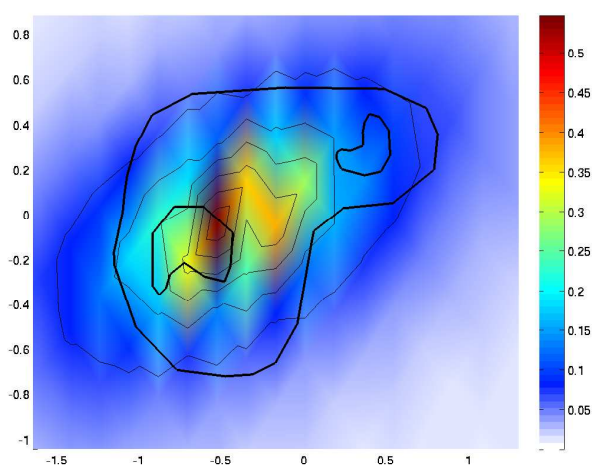

(a) $k=0.3$

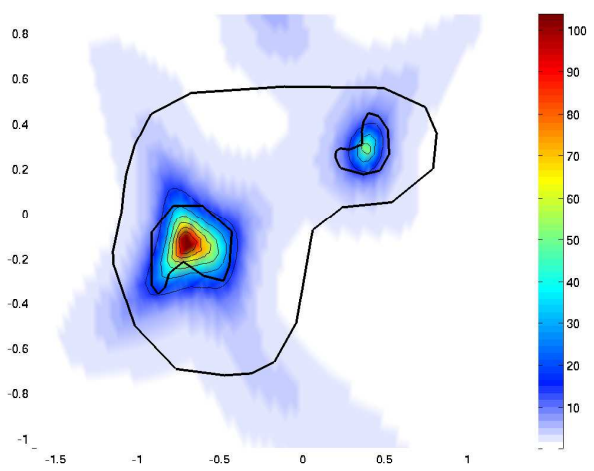

(c) $k=3$

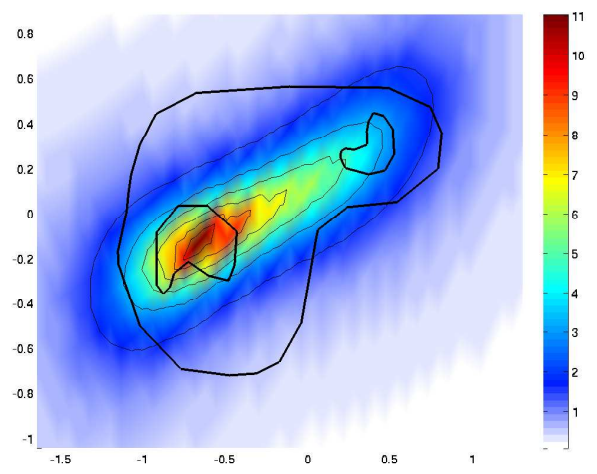

(b) $k=1$

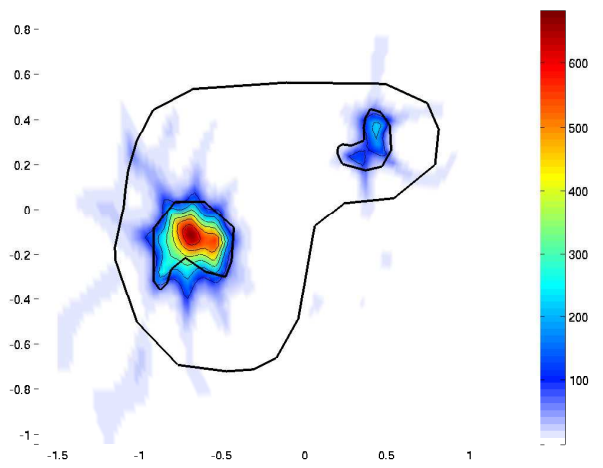

(d) $k=9$

Figure 4: Values of $w_{\left\{n_{0}, n_{1}\right\}}$ for real-valued indices and different wave numbers. 
and $4 \mathrm{~d}$.

It comes out from these tests that the method is very stable with respect to measurement errors when the wave number is fitted to the size of the defects. In these conditions, the possibly multiple connected components of the defects are properly located.

\subsection{Extension of the method: Complex-valued indices and dissociated incidence/directions of measurements}

We compute now $w_{\left\{n_{0}, n_{1}\right\}}$ from the singular values and right singular vectors for the discrete version $\mathbf{W}^{\infty}$ instead of the eigensystem for $\widetilde{W}_{\#}$, as stated in Remark 6.2.
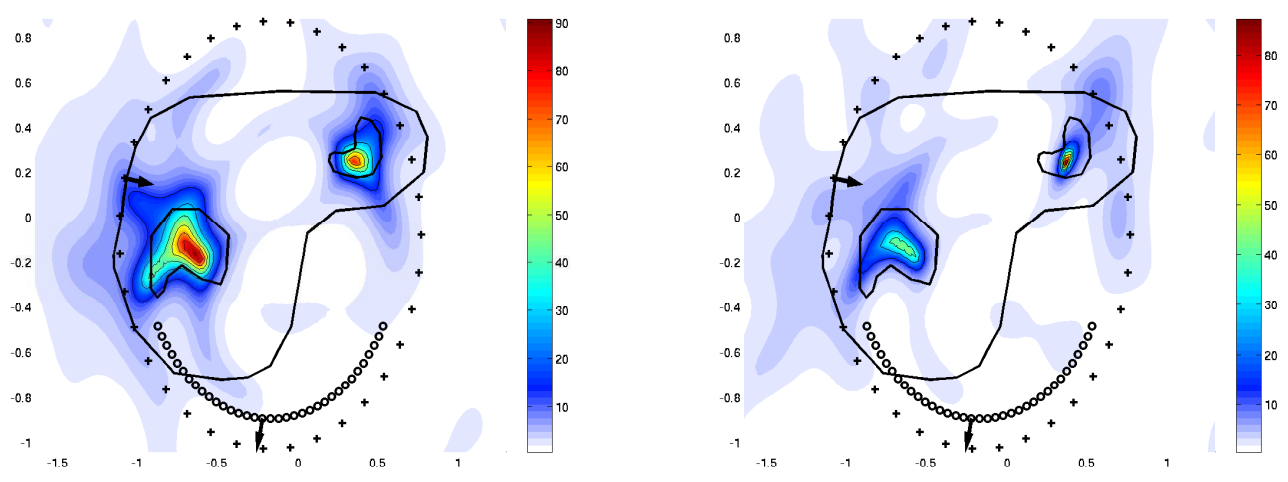

(a) $n_{0} \in[2.19,2.29]+i[0.01,0.12]$ inside $D$ and $n_{1} \in(\mathrm{b}) n_{0} \in[2.08,2.30]+i[0.00,0.15]$ inside $D$ and $n_{1} \in$ $[1.63,1.69]+i[2.04,2.12]$ inside $\Omega$ $[1.52,1.70]+i[0.10,0.24]$ inside $\Omega$
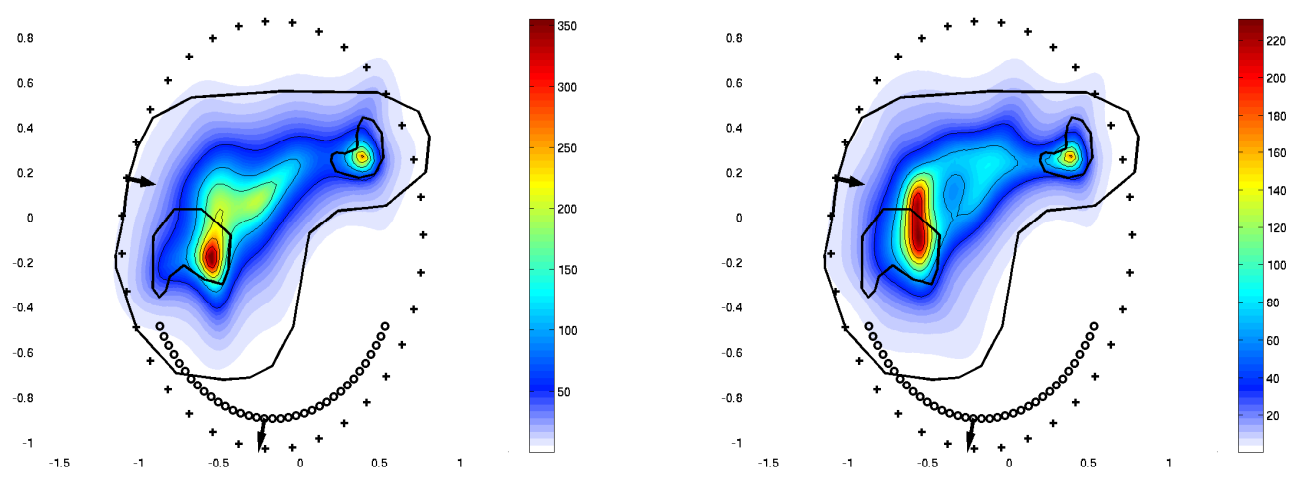

(c) $n_{0} \in[2.35,2.40]+i[2.05,2.35]$ inside $D$ and $n_{1} \in(\mathrm{d}) n_{0} \in[2.35,2.39]+i[2.05,2.29]$ inside $D$ and $n_{1} \in$ $[1.77,1.80]+i[0.11,0.31]$ inside $\Omega$ $[1.76,1.74]+i[1.91,2.04]$ inside $\Omega$

Figure 5: Values of $w_{\left\{n_{0}, n_{1}\right\}}$ for varying complex-valued indices and $\Gamma_{m} \neq S^{d-1} \neq \Gamma_{e}$.

Figure 5 presents the results obtained with a set of directions of measurements $\Gamma_{m}$ different from the incidence directions set $\Gamma_{e}$ and different complex-valued indices. 
We take $k=6, \Gamma_{e}=[0 ; 2 \pi]$ with 35 incidence directions $(+\operatorname{symbol}), \Gamma_{m}=\left[\frac{7}{6} \pi ; \frac{11}{6} \pi\right]$ with 21 measurements directions (o symbol) and $2 \%$ additional noise. In Figure 5a, we have $n_{0} \in$ $[2.19,2.29]+i[0.01,0.12]$ inside $D$ and $n_{1} \in[1.63,1.69]+i[2.04,2.12]$ inside $\Omega$. This means that the absorption is low in the background and high in the defects. The output is similar to the one in Figure 5b where $n_{0} \in[2.08,2.30]+i[0.00,0.15]$ inside $D$ and $n_{1} \in[1.52,1.70]+i[0.10,0.24]$ inside $\Omega$. Hence, if absorption of the background is low, the defects are properly located. In Figure $5 \mathrm{c}$, we have $n_{0} \in[2.35,2.40]+i[2.05,2.35]$ inside $D$ and $n_{1} \in[1.77,1.80]+i[0.11,0.31]$ inside $\Omega$. This means that the absorption is high in the background and low in the defects. The output is similar to the one in Figure $5 \mathrm{~d}$ where $n_{0} \in[2.35,2.39]+i[2.05,2.29]$ inside $D$ and $n_{1} \in[1.76,1.74]+i[1.91,2.04]$ inside $\Omega$ : A highly absorbing background significantly degrades the visual quality of the localization method, even with highly contrasting defects.

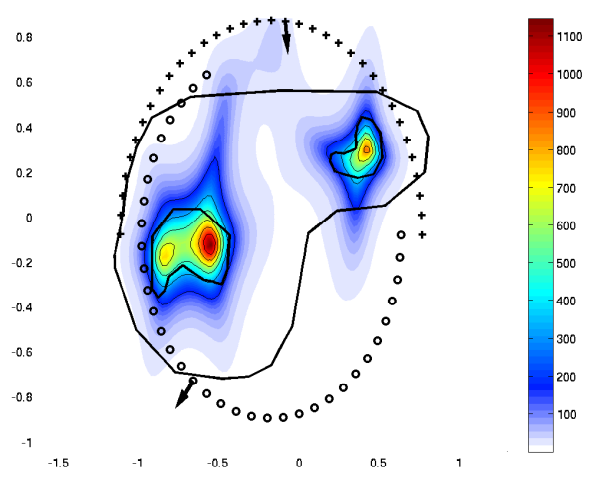

(a) $\Gamma_{m}=\left[\frac{4}{6} \pi, \frac{12}{6} \pi\right]$

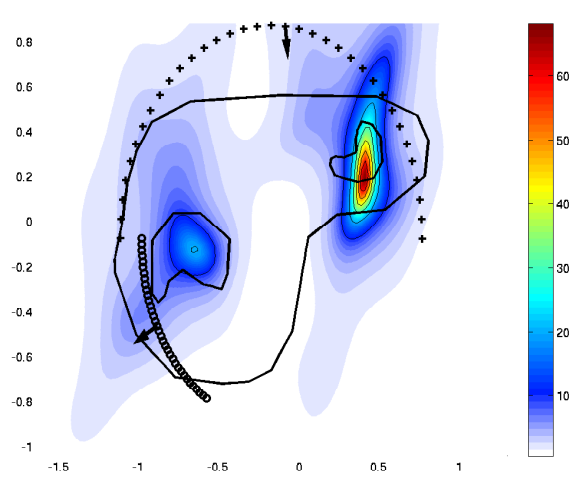

(c) $\Gamma_{m}=\left[\frac{6}{6} \pi, \frac{8}{6} \pi\right]$

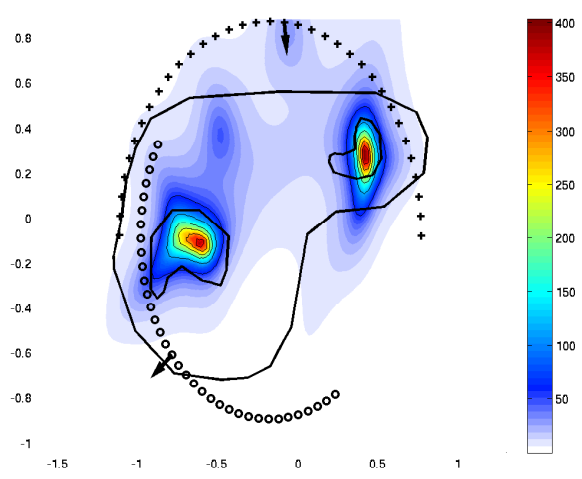

(b) $\Gamma_{m}=\left[\frac{5}{6} \pi, \frac{10}{6} \pi\right]$

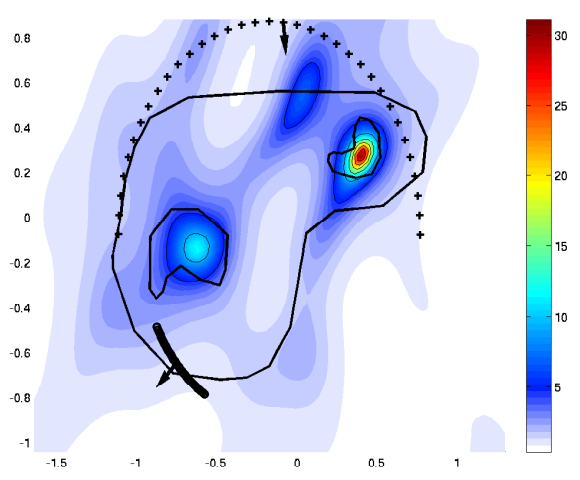

(d) $\Gamma_{m}=\left[\frac{7}{6} \pi, \frac{8}{6} \pi\right]$

Figure 6: Values of $w_{\left\{n_{0}, n_{1}\right\}}$ for varying measurements apertures with complex-valued indices.

We see in Figure 6 the results obtained by gradually reducing the measurements aperture $\Gamma_{m}$. The reference index $n_{0}$ takes values in $[1.251 .31]+i[0.09,0.18]$ inside $D$ and $n_{1}$ takes values in $[1.871 .91]+i[0.51,0.56]$ inside $\Omega$. The wave number $k$ is taken equal to 6 and we generate 35 measurements with $2 \%$ additional noise for the same number of incidence directions. 
As expected, reducing the measurements aperture, even with a fixed number of directions, degrades the quality of the reconstruction.

Finally, in realistic applications, it could happen that the reference index $n_{0}$, which is not constant, is not precisely known. Thus, we study the behaviour of the defects reconstruction method when having access only to an approximation of the reference index, or an average value, denoted by $\widetilde{n}_{0}$.

First, the exact values of the reference index $n_{0}$ are taken in $[1.241 .31]+i[0.08,0.17]$ inside $D$ and we build $n_{1}$ from $n_{0}$ as before: $n_{1}=n_{0}$ inside $D \backslash \Omega$ and $n_{1}$ takes values in [1.86 1.91] + $i[0.50,0.57]$ inside $\Omega$. We take $k$ equal to 6 and generate 35 measurements in $\left[\frac{7}{6} \pi, \frac{11}{6} \pi\right]$ with 21 incidence directions in $[0, \pi]$ and $2 \%$ additional noise. Then, we set $\widetilde{n}_{0}$ to 1 out of $D$ and to $1.2790+0.1210 i$ inside $D$, which is the average value of $n_{0}$ inside $D$.

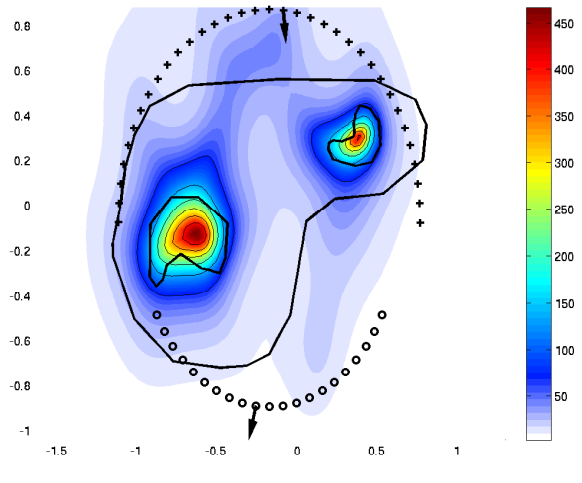

(a) averaged $n_{0}$

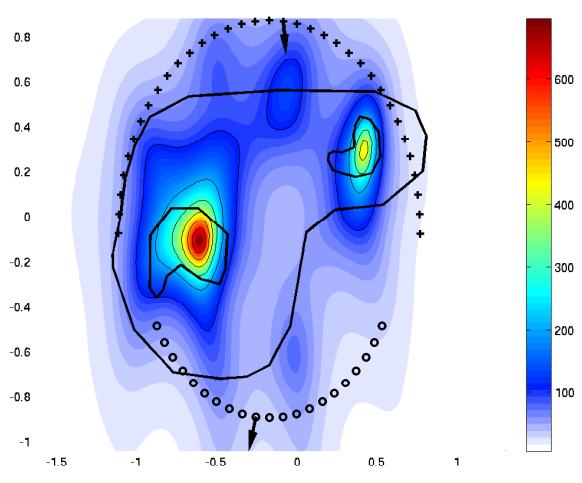

(c) $n_{0}$ averaged and shifted by $10 \%$

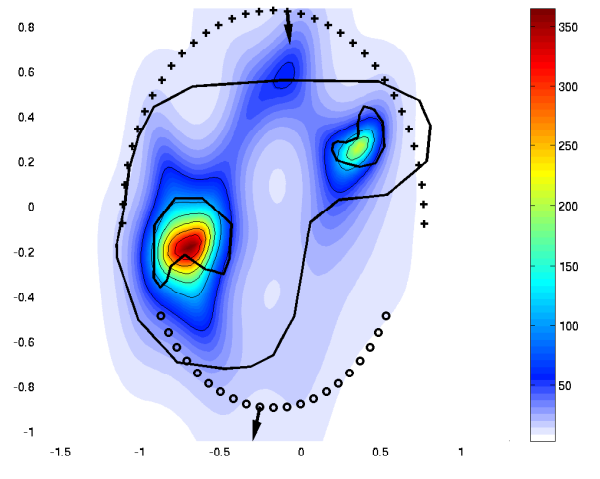

(b) $n_{0}$ with $50 \%$ additional noise

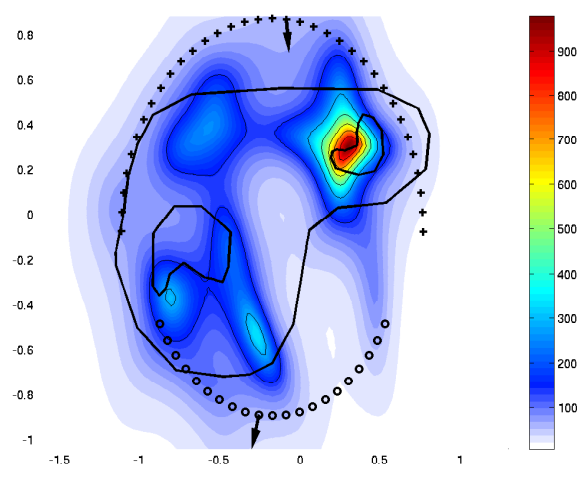

(d) $n_{0}$ averaged and shifted by $30 \%$

Figure 7: Values of $w_{\left\{n_{0}, n_{1}\right\}}$ for varying approximations of the reference index $n_{0}$.

Figure 7 a shows the plot of $w_{\left\{\widetilde{n}_{0}, n_{1}\right\}}$ built with the modified test functions $u_{\widetilde{n}_{0}}$ and where $\mathbf{W}^{\infty}$ is formed from $u_{\widetilde{n}_{0}}^{\infty}$ instead of the exact values. The visual quality of the reconstruction using an averaged reference index $\widetilde{n}_{0}$ is similar to the quality obtained with the exact values of $n_{0}$.

Figure $7 \mathrm{~b}$ shows a plot of $w_{\left\{\widetilde{n}_{0}, n_{1}\right\}}$ where $\widetilde{n}_{0}$ is $n_{0}$ with $50 \%$ uniform random noise added in 
$D$. Even with a high level of noise added to the values of the reference index, we notice that the defects are reconstructed as well as without any noise.

Finally, we study the influence of inaccuracy when considering an averaged version of the reference index. Hence, we replace the values of $n_{0}$ inside $D$ by a constant value which is an approximation of the average of the reference index inside $D$. Thus, we study the behaviour of the method in cases where only an inaccurate average of the reference index is known. Figure 7c shows an example where the values of $n_{0}$ inside $D$ are replaced by a constant value approximating the average value of $n_{0}$ inside $D$ within $10 \%$. We notice that the reconstruction is still fine. With these settings, we have to deviate from the exact average by more than $20 \%$ to ensure a significant degradation of the reconstruction's quality. An example is shown in Figure 7d, where $\widetilde{n}_{0}$ is taken equal to the average value of $n_{0}$ inside $D$ multiplied by 1.28 .

This method to reconstruct the shape of defects seems to provide good numerical results when the surrounding absorption is low. The results with high surrounding absorption might be physically interpreted as follows: The waves carrying the information about defects have to go through the absorbing inhomogeneity before, and thus loose too much amplitude to be relevant compared to the noise level. However, even with a low contrast between defects and background, as in Figure 5(d), while the shape is not reconstructed, the location of the defects is still available.

Furthermore, we notice that in the good case of low background absorption and wide measurements aperture, the method is highly insensitive to errors on the reference index. More specifically, it seems that knowing an approximation of the average value of this index is enough to detect defects in it from measurements as if the exact values of the reference index were known. This suggests a good behaviour in realistic applications.

Remark 7.1. This method has been presented in the case where the reference index is precisely known. Thus, the values $u_{n_{0}}^{\infty}$ can be computed and the test functions $u_{n_{0}}\left(\cdot, z_{i}\right)$ can be interpolated on the set of sampling points $\left\{z_{i}\right\}$. But we could assume that $u_{n_{0}}^{\infty}$ is only known from measurements, like $u_{n_{1}}^{\infty}$ is. This would be the case, for example, when monitoring moving objects. Due to the low sensitivity of this method to uncertainties about the reference index, we hope to obtain good results when $u_{n_{0}}^{\infty}$ is known through measurements. Thus, addressing the inverse problem of approximating the test functions $u_{n_{0}}\left(\cdot, z_{i}\right)$ from the (measured) values $u_{n_{0}}^{\infty}$ could extend the actual algorithm to further applications. This can be done, for instance, by a potential approach, as outlined in [25]. Indeed, the results shown in Figure 7 allow us to expect good reconstructions even with approximate test functions.

\section{Conclusion}

We have developed a simple and fast method to reconstruct the shape of defects in a (given) reference index. This method relies on the spectral data of an operator built from far-field measurements done with the actual index and near-field data computed with the reference index, rather than Green's functions. This leads to a formulation which easily fits in more general inverse problems where the near-field data are usually required at each step of an iterative process. The provided numerical examples have shown the low sensitivity of this method to uncertainties about the reference index or the measurements. This lets presume of a good behaviour in realistic applications and opens a new direction for the detection of moving objects. At last, the presented numerical evidence also encourages us to investigate the case of dissociated measurements and incidence directions. 


\section{Acknowledgments}

Support for some of the authors of this work was provided by the FRAE (Fondation de Recherche pour l'Aéronautique et l'Espace, http://www.fnrae.org/), research project IPPON.

We also want to thank the anonymous referee for bringing to our knowledge the existence of a new version of the $F_{\#}$ factorization in [20], avoiding the look for possible solutions to a transmission eigenvalue problem (see for instance [8, Theorem 2.15]).

\section{References}

\section{References}

[1] D. Colton and A. Kirsch. A simple method for solving inverse scattering problems in the resonance region. Inverse Problems, 12(4):383-393, 1996.

[2] D. Colton, M. Piana, and R. Potthast. A simple method using Morozov's discrepancy principle for solving inverse scattering problems. Inverse Problems, 13(6):1477-1493, 1997.

[3] F. Collino, M'B. Fares, and H. Haddar. On the validation of the linear sampling method in electromagnetic inverse scattering problems. Research Report 4665, INRIA, 2002.

[4] D. Colton. Inverse acoustic and electromagnetic scattering theory. In Gunther Uhlman, editor, Inside out: inverse problems and applications, volume 47 of Math. Sci. Res. Inst. Publ., pages 67-110. Cambridge Univ. Press, Cambridge, 2003.

[5] A. Kirsch. Characterization of the shape of a scattering obstacle using the spectral data of the far field operator. Inverse Problems, 14(6):1489-1512, 1998.

[6] T. Arens. Why linear sampling works. Inverse Problems, 20:163, 2004.

[7] A. Kirsch. The MUSIC algorithm and the factorization method in inverse scattering theory for inhomogeneous media. Inverse Problems, 18(4):1025-1040, 2002.

[8] A. Kirsch and N.I. Grinberg. The factorization method for inverse problems, volume 36 of Oxford Lecture Series in Mathematics and its Applications. Oxford University Press, Oxford, 2008.

[9] A. I. Nachman, L. Päivärinta, and A. Teirilä. On imaging obstacles inside inhomogeneous media. J. Funct. Anal., 252(2):490-516, 2007.

[10] N. Hyvönen. Application of the factorization method to the characterization of weak inclusions in electrical impedance tomography. Adv. in Appl. Math., 39(2):197-221, 2007.

[11] B. Gebauer. The factorization method for real elliptic problems. Z. Anal. Anwend., 25(1):81102, 2006.

[12] S.W. Doebling, C.R. Farrar, and M.B. Prime. A summary review of vibration-based damage identification methods. Shock and Vibration Digest, 30(2):91-105, 1998.

[13] I.G. Scott and C.M. Scala. A review of non-destructive testing of composite materials. NDT International, 15(2):75-86, 1982. 
[14] G. Venkov. Atkinson-Wilcox expansion theorem for inhomogeneous media. In Math. Proc. R. Ir. Acad., volume 108, pages 19-25, 2008.

[15] S. G. Mikhlin. Mathematical physics, an advanced course. North-Holland Publishing Co., Amsterdam, 1970.

[16] H. Egger, M. Hanke, C. Schneider, J. Schöberl, and S. Zaglmayr. Adjoint-based sampling methods for electromagnetic scattering. Inverse Problems, 26(7):074006, 17, 2010.

[17] L. Ségui. Sur un problème inverse en diffraction d'ondes : identificaiton de permittivités complexes d'un matériau à partir de données du champ proche. $\mathrm{PhD}$ thesis, Université Toulouse III, 2000.

[18] C. Hazard and K. Ramdani. Selective acoustic focusing using time-harmonic reversal mirrors. SIAM Journal on Applied Mathematics, 64(3):1057-1076, 2004.

[19] T. Kato. Perturbation theory for linear operators. Classics in Mathematics. Springer-Verlag, Berlin, 1995. Reprint of the 1980 edition.

[20] A. Lechleiter. The factorization method is independent of transmission eigenvalues. Inverse Probl. Imag, 3:123-138, 2009.

[21] D. Colton and R. Kress. Inverse acoustic and electromagnetic scattering theory, volume 93 of Applied Mathematical Sciences. Springer-Verlag, Berlin, second edition, 1998.

[22] A. Kirsch. An introduction to the mathematical theory of inverse problems, volume 120 of Applied Mathematical Sciences. Springer-Verlag, New York, 1996.

[23] P.D. Hislop and I.M. Sigal. Introduction to spectral theory: With applications to Schrödinger operators. Springer, 1996.

[24] B. Dah. Sur la modélisation de milieux fictifs absorbants de type couches de Bérenger. $\mathrm{PhD}$ thesis, Université Toulouse III, 2001.

[25] D. Colton and R. Kress. Using fundamental solutions in inverse scattering. Inverse Problems, 22:R49-R66, 2006. 